\title{
Evaluación de necesidades paliativas en el paciente con enfermedad renal crónica en hemodiálisis
}

\author{
Ian Blanco Mavillard1,5, Juan Pablo Leiva Santos², Gregorio Bolaños Herrezuelo³, Catalina Jaume Riutort ${ }^{3}$, \\ Joana Maria Julià Móra², Miguel Ángel Rodríguez Calero ${ }^{4,5}$ \\ ${ }^{1}$ Unidad de calidad, docencia e investigación del Hospital de Manacor. Mallorca. ${ }^{2}$ Equipo de soporte hospitalario \\ de atención paliativa, Hospital de Manacor. Mallorca. ${ }^{3}$ Unidad de hemodiálisis. Hospital de Manacor. Mallorca. \\ ${ }^{4}$ Coordinador de investigación en cuidados del Servicio de Salud de las Islas Baleares. ${ }^{5}$ Profesor asociado del \\ departamento de Enfermería y Fisioterapia de la Universitat de les Illes Balears. Palma de Mallorca. España
}

\section{Resumen}

Introducción: Los cuidados paliativos aportan una atención necesaria que promueve una respuesta integral y coordinada del sistema sanitario respetando la autonomía y valores de los pacientes en situación de final de vida.

Objetivos: Determinar la prevalencia de necesidad de cuidados paliativos en la unidad de hemodiálisis de nuestro centro mediante el instrumento validado NECPAL CCOMSS-ICO e identificar la situación de complejidad en pacientes con necesidades paliativas usando la herramienta de IDC-Pal.

Material y Método: Estudio observacional transversal. Tres enfermeras con formación en cuidados paliativos $y$ en el uso de los cuestionarios NECPAL CCOMS-ICO y el IDC-Pal evaluaron de forma independiente a todos los pacientes de la unidad de crónicos de hemodiálisis. Las evaluaciones fueron realizadas entre los días 6 y 10 de marzo. Las discrepancias se resolvieron consensuando el resultado final entre las tres profesionales.

Resultados: Se analizaron a 50 pacientes, con una edad media de 68.24. El perfil de paciente más frecuente fue aquel con un tiempo de permanencia en hemodiálisis de 48,8 meses de media. La enfermedad renal primaria y comorbilidad más prevalente fueron la nefropatía diabética (26\%) y la hipertensión arterial

Correspondencia:
Ian Blanco Mavillard
Enfermero referente de Investigación
Unidad de Calidad, Docencia e Investigación
del Hospital de Manacor
Ctra. de Alcudia- Palma, s/n.07500 Manacor. España
E-mail: ianblanco7@gmail.com

(56\%) respectivamente. Se identificaron 20 pacientes con necesidad de atención paliativa (40\%). Respecto a la evaluación del IDC-Pal, se obtuvieron 19 pacientes en situación de complejidad y 1 en situación de no complejidad.

Conclusiones: Las necesidades paliativas complejas sugieren la necesidad de realizar evaluaciones periódicas en las unidades de hemodiálisis, así como un enfoque asistencial multidisciplinar para dar respuesta a las necesidades identificadas.

PALABRAS CLAVE: hemodiálisis; cuidados paliativos; necesidad paliativa; integración.

\section{Palliative needs assessment in patients with chronic kidney disease on hemodialysis}

\section{Abstract}

Introduction: The palliative care provides a necessary attention that promotes a comprehensive response and coordination of the health system respecting the autonomy and values of patients in the end-of-life situation.

Aim: To determine the prevalence of the need for palliative care in the hemodialysis unit of our center using the validated instrument NECPAL CCOMSSICO and to identify the complexity situation in patients with palliative needs using IDC-Pal.

Material and Method: Cross-sectional observational study. Three nurses trained in palliative care and in the use of the NECPAL CCOMS-ICO and IDC-Pal questionnaires independently assessed all patients in 
the chronic hemodialysis unit. The evaluations were carried out between the 6th and 10th of March. The discrepancies were resolved agreeing the final result among the three professionals.

Results: 50 patients were analysed, with a mean age of 68.24. The most frequent patient profile was with a permanence time in hemodialysis of 48.8 months of means. The most prevalent renal disease and comorbidity were diabetic nephropathy $(26 \%)$ and hypertension $(56 \%)$, respectively. 20 patients with palliative care $(40 \%)$ were identified. Regarding the evaluation of the IDC-Pal, 19 patients were obtained in a situation of complexity and 1 in situation of noncomplexity.

Conclusions: Complex palliative needs suggest the need for periodic evaluations in hemodialysis units, as well as a multidisciplinary care approach to respond to identified needs.

KEYWORDS: hemodialysis; palliative care; palliative needs; integration.

\section{Introducción}

Según La Estrategia Nacional de Cuidados Paliativos, los pacientes con cáncer y enfermedades crónicas evolutivas de cualquier edad que se encuentren en situación avanzada deben beneficiarse de los valores y principios de los Cuidados Paliativos ${ }^{1 .}$ Estos aportan una atención necesaria que promueve una respuesta integral y coordinada del sistema sanitario respetando la autonomía y valores de los pacientes en situación de final de vida.

La integración de los cuidados paliativos (CP) implica articular los aspectos administrativos, organizativos, clínicos y de servicios para asegurar la continuidad de la atención entre todos los profesionales involucrados en la red de cuidados de los pacientes que necesitan una atención paliativa ${ }^{2}$. Su objetivo no es prolongar la supervivencia del paciente, sino conseguir un manejo conservador de la enfermedad. Esto es necesario para preservar la calidad de vida, además de ofrecer un soporte adecuado al paciente y su familia en el proceso de final de vida, en colaboración con todos los cuidadores. El proceso asistencial integrado de cuidados paliativos incorpora el concepto de complejidad para establecer una correcta adecuación de las diferentes intervenciones en la atención de pacientes en situación terminal ${ }^{3}$.
Aproximadamente un $75 \%$ de la población de nuestro país morirá a causa de una enfermedad crónica ${ }^{4}$. Se calcula que cada año mueren 380.000 personas en España, entre el $50-60 \%$ de estas personas podrían necesitar $\mathrm{CP}^{5}$. Se han utilizado diferentes sistemas para calcular la prevalencia de necesidades paliativa, a partir de la extracción de diagnósticos de causa de muerte definidas por McNamara ${ }^{6}$ y mediante instrumentos para la detección de CP en enfermos en situación de cronicidad $^{7,8}$. La prevalencia global de los pacientes con Enfermedad renal crónica avanzada (ERCA) en estadio 5 sigue incrementándose, especialmente entre los pacientes de edad avanzada. Por otro lado, la epidemiología de los pacientes con ERCA ha cambiado considerablemente debido a un acceso menos restrictivo a la diálisis, especialmente en los países desarrollados?. Esto se acentúa, observando un crecimiento en el número de pacientes mayores de 75 años en tratamiento renal sustitutivo (TRS), siendo del $17,7 \%$ en 1992 al $24,5 \%$ en $2012^{10}$.

En la actualidad, se identifica como un elemento fundamental conocer las necesidades de atención paliativa de los pacientes renales para el diseño de políticas sanitarias dirigidas a la cronicidad adaptadas a sus necesidades y preferencias. ${ }^{11}$ Por lo tanto, los objetivos de este estudio son determinar la prevalencia de necesidad de cuidados paliativos en la unidad de hemodiálisis de nuestro centro mediante el instrumento validado NECPAL CCOMSS-ICO e identificar la complejidad en pacientes con necesidades paliativas usando la herramienta de IDC-Pal.

\section{Material y Método}

Estudio observacional descriptivo transversal realizado en la unidad de hemodiálisis del Hospital de Manacor, Mallorca. Se seleccionó como población de estudio a todos los pacientes de la unidad de hemodiálisis $(n=50)$, excluyendo a todos los pacientes menores de 18 años. Se emplearon los cuestionarios NECPAL CCOMS-ICO(4) (en adelante NECPAL), validado en el contexto español y utilizado para detectar necesidades en la atención paliativa de la población general, ${ }^{12}$ y el IDC-Pal, ${ }^{13}$ herramienta diagnóstica y clasificatoria de situaciones o elementos de complejidad susceptibles de aparecer en pacientes con necesidad de cuidados paliativos. IDC-Pal solo se utilizó en aquellos pacientes con necesidad actual de cuidados paliativos (NECPAL+). Se recogieron las variables sobre las características demográficas de los pacientes (edad, sexo, meses de permanencia en TRS, tipo de TRS, tipo de acceso 
vascular, motivo de la enfermedad renal primaria, comorbilidad, índice de Charlson, e inclusión actual en programa de cuidados paliativos), variables relacionadas con el instrumento NECPAL (Pregunta sorpresa, demanda de atención paliativa, y necesidad paliativa) y variables relacionadas con el cuestionario IDC-Pal (situación de complejidad e intervención de los recursos avanzados).

Las evaluaciones fueron realizadas durante los días del 6 al 10 de marzo de 2017, por tres enfermeras de la unidad de hemodiálisis con formación en CP y en el uso de ambas herramientas. Cada una de las enfermeras evaluó independientemente a cada paciente. Las discrepancias se resolvieron consensuado el resultado final entre los evaluadores. Se realizó un análisis descriptivo de la información. Las variables cuantitativas se representan mediante media y desviación estándar, y las cualitativas mediante tablas de frecuencia. El trabajo recibió la aprobación de las comisiones de soporte para la atención paliativa y de investigación y ética del Hospital de Manacor.

\section{Resultados}

\section{Características de los pacientes}

Se analizaron a 50 pacientes, 18 mujeres y 32 hombres, con una edad media de $68.24 \pm 14,137$. Las características de los pacientes se muestran en la tabla 1. El perfil de paciente más frecuente en nuestra unidad era el de mayor de 65 años, con una permanencia de tiempo en hemodiálisis de 48,8 meses $\pm 45,8$ meses. La enfermedad renal primaria y comorbilidad más prevalente en la unidad eran la nefropatía diabética (38\%) y la hipertensión arterial (56\%) respectivamente. El tratamiento renal sustitutivo más empleado era la hemodiálisis (54\%) con un tiempo medio de 12 horas semanales $(62 \%)$.

\section{Detección de la necesidad de atención paliativa}

Los resultados obtenidos de las evaluaciones del instrumento NECPAL, pregunta sorpresa, demanda y necesidad de atención paliativa; y del IDC-Pal, situación de complejidad y utilización de recursos avanzados se muestran en la tabla 2. Se obtuvo un $40 \%$ de prevalencia en el NECPAL+, es decir, se identificó a 20 pacientes en situación de enfermedad avanzada-terminal. Se valoró que 19 pacientes (38\%) requieren de cuidados paliativos, de los cuales 12 (24\%) están incluidos actualmente en el programa de CPR del centro. Respecto a los resultados del IDC-Pal, se obtuvo que 19
Tabla 1. Características demográficas.

\begin{tabular}{|c|c|}
\hline $\mathbf{N}$ & 50 \\
\hline Pérdidas & 0 \\
\hline Edad & $68.24 \pm 14,137$ \\
\hline \multicolumn{2}{|l|}{ Sexo } \\
\hline Hombre & $32(64 \%)$ \\
\hline Mujer & $18(36 \%)$ \\
\hline Tiempo permanencia en Hemodiálisis & $48,78 \pm 45,757$ \\
\hline \multicolumn{2}{|l|}{ Trasplante renal previo } \\
\hline No & $45(90 \%)$ \\
\hline $\mathrm{Si}$ & $5(10 \%)$ \\
\hline \multicolumn{2}{|l|}{ Enfermedad renal primaria } \\
\hline Nefropatía diabética & $13(26 \%)$ \\
\hline Nefropatía vascular & $4(8 \%)$ \\
\hline Glomerulonefritis & $3(6 \%)$ \\
\hline Neoplasia & $3(6 \%)$ \\
\hline Enfermedad poliquística & $2(4 \%)$ \\
\hline No filiada & $7(14 \%)$ \\
\hline Otras causas & $8(16 \%)$ \\
\hline $\begin{array}{l}\text { Pielonefritis/nefropatía } \\
\text { intersticial crónica }\end{array}$ & $4(8 \%)$ \\
\hline $\begin{array}{l}\text { Causa mixta } \\
\text { (nefropatía diabética y vascular) }\end{array}$ & $6(12 \%)$ \\
\hline \multicolumn{2}{|l|}{ Comorbilidad } \\
\hline Hipertensión arterial & $28(56 \%)$ \\
\hline Isquemia & $2(4 \%)$ \\
\hline Demencia & $2(4 \%)$ \\
\hline Insuficiencia respiratoria & $1(2 \%)$ \\
\hline Insuficiencia cardiaca & $3(6 \%)$ \\
\hline Ninguna & $14(28 \%)$ \\
\hline Índice de Charlson (Mediana) & 8 \\
\hline \multicolumn{2}{|l|}{ Tratamiento renal sustitutivo } \\
\hline Hemodiálisis convencional (HD) & $27(54 \%)$ \\
\hline Hemodiafiltración en línea (HDFOL) & $23(46 \%)$ \\
\hline Tiempo medio del tratamiento semanal & 11 y $43 \min \pm 39 \min$ \\
\hline $10 \mathrm{~h}$ y $30 \mathrm{~min}$ & $7(14 \%)$ \\
\hline $11 \mathrm{~h}$ y $15 \mathrm{~min}$ & $9(18 \%)$ \\
\hline $12 \mathrm{~h}$ & $31(62 \%)$ \\
\hline $12 \mathrm{~h}$ y $45 \mathrm{~min}$ & $2(4 \%)$ \\
\hline $13 \mathrm{~h}$ y $30 \mathrm{~min}$ & $1(2 \%)$ \\
\hline \multicolumn{2}{|l|}{ Acceso vascular } \\
\hline Catéter venoso central (CVC) & $29(58 \%)$ \\
\hline Fistula arteriovenosa (FAV) & $19(38 \%)$ \\
\hline FAV / CVC & $1(2 \%)$ \\
\hline Fistula arteriovenosa protésica (PTFEE) & $1(2 \%)$ \\
\hline
\end{tabular}

pacientes se encontraban en situación de complejidad, de los cuales 12 pacientes $(63,2 \%)$ utilizan recursos avanzados del sistema sanitario, como material ortopédico, oxigenoterapia, aerosolterapia, bombas de perfusión, material fungible, fármacos de uso hospitalario, uso reiterado de los dispositivos y servicios de urgencia, dificultad en la coordinación de traslados, ingresos programados etc. 
Tabla 2. Detección de necesidad de atención paliativa.

\begin{tabular}{ll}
\hline NECPAL CCOMS-ICO & \\
\hline $\begin{array}{l}\text { Pregunta sorpresa ¿Le sorprendería que este } \\
\text { pacientese muriera en los próximos } 12 \text { meses? }\end{array}$ & 50 \\
No (Necpal +) & $20(40 \%)$ \\
Si (Necpal -) & $30(60 \%)$ \\
Demanda de necesidad paliativa & \\
No & $45(90 \%)$ \\
Si & $5(10 \%)$ \\
Necesidad actual de atención paliativa & \\
No & $31(62 \%)$ \\
Si & $19(38 \%)$ \\
Inclusión a programa de cuidados paliativos & \\
No & $38(76 \%)$ \\
Si & $12(24 \%)$ \\
\hline IDC-Pal & \\
\hline Situación de complejidad & \\
No complejo & \\
Complejo & \\
Altamente complejo & \\
Utilización de recursos avanzados & $12(63,8 \%)$ \\
Si & 0 \\
\hline
\end{tabular}

\section{Discusión}

Este estudio pretende sentar las bases metodológicas que permita la evaluación conjunta de dichas necesidades con el objetivo de priorizar la atención paliativa en pacientes con enfermedad renal crónica en estadio avanzado. El planteamiento inicial de este estudio permitió determinar la prevalencia de pacientes con ERCA en estadio $V$ que requieren de $C P$ identificando la situación de complejidad. En la actualidad, existen estudios que emplean instrumentos para su diagnóstico de manera independiente ${ }^{13,14}$.

La prevalencia obtenida es similar a la descrita en entornos similares de nuestro país, ${ }^{15}$ aunque superior a otros estudios a nivel internacional ${ }^{16,17}$. Esto puede explicarse debido al número de evaluadores por pacientes de los estudios mencionados anteriormente, siendo un único evaluador el que realizaba la identificación. 16,17 Estudios recientes recomiendan para el uso del NECPAL estimaciones por más de un profesional, apoyando la evaluación consensuada en equipo, valor clave en la atención paliativa ${ }^{4,12}$. Conocer la necesidad de CP y el uso de herramientas de forma sistematizada puede ser de gran ayuda a la toma de decisiones por parte del equipo de salud. La identificación temprana es prioritaria, ya que nos permite entablar de forma temprana conversaciones con el paciente y su familia sobre la necesidad de cuidados y el uso de recursos sanitarios, así como las preferencias en caso de aparecer situaciones agudas o de difícil manejo. La inclusión del paciente en el programa de cuidados paliativos renales (CPR) puede ayudarnos a planificar de manera adecuada el soporte de los cuidados avanzados a la asistencia de pacientes frágiles con una esperanza de vida inferior a 1 año o con múltiples comorbilidades significativas para adecuar el soporte dialítico enfatizando el control de síntomas frente a la eficacia analítica del TRS. Estos cuidados promueven una respuesta integral, orientada a dar soporte paliativo y maximizar el bienestar del paciente y de la familia proporcionando una atención menos agresiva a pacientes en situación terminal que respete su autonomía y valores ${ }^{18-20}$. El trabajo en equipo es de vital importancia para conseguir un manejo eficaz de las diferentes situaciones clínicas que puedan ocurrir. Esto se puede relacionar directamente a la satisfacción del paciente y/o familia y al coste de la atención sanitaria.

En cuanto a la validez externa de los cuestionarios, encontramos que el instrumento NECPAL ${ }^{21}$ se ha mostrado muy consistente para determinar la necesidad actual de atención paliativa en el paciente renal, siendo una herramienta de uso sencillo y de fácil interpretación. Sin embargo, debemos señalar ciertas limitaciones del IDC-Pal para la contextualización en el paciente renal. Este instrumento de evaluación permite identificar situaciones de alta complejidad en pacientes crónicos en fase terminal o de últimos días. Cualquier paciente renal crónico en tratamiento de hemodiálisis ambulatoria clasificaría en situación de complejidad, por esta razón se recomienda su evaluación siempre que tenga criterios de necesidad paliativa.

La atención a la cronicidad avanzada es actualmente uno de los principales desafíos que nos enfrentamos los profesionales del sistema sanitario. Son necesarios nuevos estudios con mayor número de pacientes y contextos para conocer en profundidad la situación real de las necesidades de CP en hemodiálisis en nuestro país.

En conclusión, la prevalencia de necesidades paliativas complejas sugiere la necesidad de realizar evaluaciones periódicas mediante la utilización de instrumentos como NECPAL e IDC-Pal, lo cual podría ser extensivo a otras unidades similares en nuestro entorno. Un enfo- 
que asistencial multidisciplinar, que integre los cuidados paliativos podría dar respuesta a las necesidades identificadas respetando el principio ético de justicia en la atención a los enfermos en la etapa final de la vida.

\section{Conflicto de intereses}

Los autores declaran que no existen conflictos de intereses.

\section{Agradecimientos}

A todos los compañeros de diálisis del Hospital de Manacor por su colaboración y dedicación por visibilizar los cuidados paliativos renales y especialmente a todos nuestros pacientes, sin ellos nuestras iniciativas no cobrarían sentido.

Recibido: 26 abril 2017

Revisado: 5 mayo 2017

Modificado: 15 agosto 2017

Aceptado: 17 agosto 2017

\section{Bibliografía}

1. Ministerio de Sanidad y Consumo. Estrategia en cuidados paliativos del Sistema Nacional de Salud. 2007;1-161. [Consultado 18 Marzo 2017]. Disponible en: http://www.aepcp.net/arc/estrategiaCuidadosPaliativos.pdf\%5Cnhttp://publicacionesoficiales.boe.es

2. Siouta N, van Beek K, Preston N, Hasselaar J, Hughes $S$, Payne $S$, et al. Towards integration of paIliative care in patients with chronic heart failure and chronic obstructive pulmonary disease: a systematic literature review of European guidelines and pathways. BMC Palliat Care. 2016;15(1):18. Disponible en: http://www.pubmedcentral.nih.gov/ articlerender.fcgi?artid=4752742\&tool=pmcentrez\&rendertype $=$ abstract

3. Aldridge MD, Hasselaar J, Garralda $E$, van der Eerden M, Stevenson D, McKendrick K, et al. Education, implementation, and policy barriers to greater integration of palliative care: A literature review. Palliat Med. 2015;30(3):224-39.

4. Gómez-Batiste $X$, Martínez-Muñoz M, Blay C, Amblàs J, Vila L, Costa X, et al. Identifying patients with chronic conditions in need of palliative care in the general population: development of the NECPAL tool and preliminary prevalence rates in Catalonia. BMJ Support Palliat Care. 2013;3(3):300-8. Disponible en: http://spcare.bmj.com/content/early/ 2012/12/14/bmjspcare-2012-000211

5. Garralda E, Hasselaar J, Carrasco JM, Van Beek K, Siouta N, Csikos A, et al. Integrated palliative care in the Spanish context: a systematic review of the literature. BMC Palliat Care. 2016;15(1):49. Disponible en: http://www.ncbi.nlm.nih.gov/pubmed /27177608\%5Cnhttp://www.pubmedcentral.nih. gov/articlerender.fcgi?artid=PMC4865984

6. McNamara B, Rosenwax L, Holman C. A method for definingand estimating the palliative care population. J pain Sympt Manag. 2006;32:5-12.

7. McGrath S, Foote Gargis D, Frith $\mathrm{H}$, Hall Michael W. Cost Effectiveness of a Palliative Care Program in a Rural Community Hospital. Nurs Econ. 2013;31(4):176-83. Disponible en: http://search. ebscohost.com/login. aspx?direct=true $\& \mathrm{db}=\mathrm{ccm} \& A$ $\mathrm{N}=107964016 \&$ site=ehost-live

8. Highet G, Crawford D, Murray S, Boyd K. Development and evaluation of the Supportive and Palliative Care Indicators Tool (SPICT): a mixed-methods study. BMJ Support Palliat Care. 2013;4:285-90. Disponible en: http://www.ncbi. nlm.nih.gov/pubmed/24644193

9. Brown MA, Crail SM. ANZSN Renal Supportive Care Guidelines 2013 The often difficult decision of which patients will benefit from dialysis. Nephrology. 2013;18:401-54.

10. KwokAOL,Yuen S,Yong DS,Tse DMW.The Symptoms Prevalence, Medical Interventions, and Health Care Service Needs for Patients With End-Stage Renal Disease in a Renal Palliative Care Program. Am J Hosp Palliat Med. 2016;33(10):952-8. Consultado en: http://ajh.sagepub.com/cgi/doi/10. $1177 / 1049909115598930$ 
11. Gómez-Batiste X, Pascual A, Espinosa J, Caja C. Diseño, implementación y evaluación de programas públicos de cuidados paliativos. Med Clin (Barc). 2010;135(4):179-85.

12. Rodríguez-Calero MÁ, Julià-Mora JM, Prieto-Alomar A. Detección de necesidad de atención paliativa en una unidad de hospitalización de agudos. Estudio piloto. Enferm Clin. $2016 ; 26(4): 238-42$. Disponible en: http://dx.doi.org/10.1016/j.enfcli. 2015.11.004

13. Martín-Roselló M, Fernández-López M, VidalEspaña F, Sanz-Amores R. Development of a Method of Diagnosing Complexity in Palliative Care. Palliat Med. 2014;28(6):761-2.

14. Martín-Utrilla S, Villasana-Sarabia A, GallartGómez $P$, Mancheño-Álvaro A. Diagnóstico de la complejidad de la atención paliativa en hospitalización a domicilio versus hospitalización convencional. Therapeía. 2016;8:31-45.

15. Jimenez M, Polanco S, Davin E, Sanchidrian S. Frailty, surprise question and mortality in a hemodilaysis cohort question and mortality in a hemodialysis cohort. Nephrol Dial Transpl. 2016;31:i553.

16. Cohen L, Ruthazer R, Moss A, Al. E. Predicting six-month mortality for patients who are on maintenance hemodialysis. Clin J Am Soc Nephrol. 2010;5:72-9.
17. Moss A, Ganjoo J, Sharma S, Al. E. Utility of the "surprise" question to identify dialysis patients with high mortality. Clin J Am Soc Nephrol. 2008;3:1379-84.

18. Sociedad Españolade Cuidadospaliativos.Guíadecuidados paliativos. Secpal.2002;1-52. Disponible en: http://www.secpal.com/guiacp/guiacp.pdf \%5Cnhttp://cmvinalo.webs.ull.es/docencia/Posgrado /8-CANCER Y CUIDADOS-PALIATIV0S/guiacp. pdf

19. Parikh R, Kirch R, Smith T, Al. E. Early specialty palliative care - translating data in oncology into practice. N Engl J Med. 2013;369:2347-51.

20. Temel J, Greer J, Muzikansky A, AI E. Early palliative care for patients with metastatic non-small-cell lung cancer. N Engl J Med. 2010;363:733-42.

21. Gómez-Batiste X, Martínez-Muñoz M, Blay C, Amblàs J, Vila L, Costa X. Identificación de personas con enfermedades crónicas avanzadas y necesidad de atencion paliativa en servicios sanitarios y sociales: elaboración del instrumento NECPAL CCOMS-ICO. Med Clin (Barc). 2013;140(6):241-5. 